\title{
Rhino Cerebral Mucormycosis with Post Surgical Complications in Diabetes: A Case Report
}

\author{
*N Mahmood ${ }^{1}$, F A Quraishi² ${ }^{2}$ S M H Shahid ${ }^{3}$ \\ S M K Majumder ${ }^{4}$, H Haque ${ }^{5}$, A K M A Haque ${ }^{6}$, Z S Shahid ${ }^{7}$ \\ ${ }^{1}$ Dr. Nazneen Mahmood, Associate Professor, Department of Nephrology, AKMMC \\ ${ }^{2}$ Prof (Dr) Firoz Ahmed Quraishi, Professor and Head, Department of Neurology, AKMMC \\ ${ }^{3}$ Col.(Dr) S M Hossain Shahid, Consultant, Intensive Care Unit, AKMMCH \\ ${ }^{4}$ Prof.(Dr) SM Khorshed Majumder, Professor and Head Department of ENT, Holy Family Red Crescent \\ Medical College \& Hospital, Dhaka \\ ${ }^{5}$ Dr. Harisul Haque, Associate Professor, Department of Cardiology, BSMMU \\ ${ }^{6} \mathrm{Dr}$. A K M Akramul Haque, Associate Professor, National Institute of Diseases of Chest and Hospital \\ Mahakhali, Dhaka \\ ${ }^{7}$ Dr Zakia Sultana Shahid, Associate Professor and Head, Department of Ophthalmologys, AKMMC
}

*Corresponding author

Date of submission: 10 July 2015 Date of acceptance: 21 December 2016

\begin{abstract}
Rhinocerebral mucormycosis is a rare life-threatening infection caused by fungi from the order Mucorales. The disease occurs mostly in association with diabetic ketoacidosis. Because of its rapid progression and high mortality, early recognition and aggressive treatment offer the only chance to increase the survival rate. A case of rhinocerebral mucormycosis in a diabetic male patient presenting as post surgical complication is reported. The clincal manifestations, diagnosis, and treatment of rhinocerebral mucormycosis are discussed.
\end{abstract}

Key Words: Rhinocerebral mucormycosis, Diabetes mellitus, Meningitis, Amphotericin B.

\section{Introduction}

RCM (Rhinocerebral mucormycosis) is also known as phacomycosis or zygomycosis, is a rare fatal opportunistic infection caused by the family of mucoraceae (Mucor, Rhizomucor, Rhizopus, Absidia species) that are commonly found in soil and among decaying vegetation ${ }^{1,2}$. RCM is usually seen in diabetics, especially in ketoacidosis, but may also appear in healthy people $^{3}$. Several clinical forms are described such as rhino-orbital-cerebral, pulmonary, sdisseminated, cutaneous, and gastrointestinal. Rhino-orbital-cerebral is the most common form in diabetic patients ${ }^{4}$. Initially majority of patients complain about the typical symptoms of acute bacterial rhinosinusitis but they deteriorate rapidly and do not respond to conventional treatment, such patients are usually associated with diabetic ketoacidosis (DKA). Early CT imaging is usually unimpressive. Direct extension and dissemination via blood leads to orbital and intracranial complications1. The diagnosis is confirmed histologically and culture is used to identify the specific species. The treatment of mucormycosis includes aggressive surgical debridement and systemic antifungal therapy. Early diagnosis and prompt initiation of treatment is essential for a successful outcome. Disease extending beyond the sinus mucosa and associated DKA on initial presentation are poor prognostic indicators5. Newer drugs like Posaconazole and micafungin are being tried with Amphotericin B with variable results. A post surgical case of RCM are discussed which had a variable clinical course following standard treatment protocol. 


\section{Case Report}

Mr. Md. Nurul Islam, 42 years, male, diabetic for 15 years, hypertensive for 10 years, non asthmetic patient got admitted into Anwer Khan Modern Medical College Hospital(AKMMCH) on 2.815 at 3.30 AM with the complaints of high grade fever for 6 days and vomiting for several episodes.

Initially the patient was presented with 6 months history of right sided temporal headache and blurring of vision in right eye. Probably this incidence was happened in November 2014, when he went to a dental surgeon for extraction of third molar tooth. After tooth extraction pain did not resolve. His blood sugar was poorly controlled. HbA1C was $9.5 \%$. The patient underwent FESS(Functional Endoscopic Sinus Surgery) surgery on April 2015 in Bangladesh Apollo Hospital. Histo-pathology report of nasal tissue wassections from specimen show multiple fragments of chronically inflamed, oedematous respiratory mucosa, dense, inflammatory fibrinous exudate and diffuse infiltration of acute and chronic inflammatory cells. Presence of large tangles of fungal hyphae(PAS positive) admixed with dense fragments of inflammatory fibrinous exudate are also noted. These fungal hyphae are non-septate, irregularly wide showing right angle branching. Histological diagnosis was Mucor mycosis and the patient was treated with Amphotericin B.

The patient developed complete loss of vision on 5th June 2015. Then the patient was referred to ENT Department and they planned for FESS surgery again and was started Liposomal Amphotericin B after hospitalisation. Later on patient developed increase headache and vomiting and FESS surgery was not possible due to hyponatraemia( $\mathrm{Na}$ level was $112 \mathrm{mmol} / \mathrm{L})$. MRI brain with contrast done at that time. It showed locally aggressive lesion consistent with mucormycosis in the right orbital apex, right maxillary ethmoidal sinus, right nasal cavity, pterygopontine fossa, lesser wings of sphenoid, optic foramina involving optic nerve, superior orbital fissure involving cranial nerves and extension into right middle cranial fossa involving meninges over cavernous sinus.Then after diagnosing Rhino orbito sphenoidal mucormycosis patient underwent extensive debridement with right orbital exenteration on 18.6.2015. Histopathology report of tissue from right maxillary antrum and pterygopalatine fossa showed chronic inflammation and foci of necrosis with fungal hyphae. This time the patient was treated with Posaconazole.

In the mean time on 6th July 2015, patient again complain of high grade fever and headache since 5 days with $\mathrm{H} / \mathrm{O}$ vomiting for 2 days and bilateral leg swelling for 2 days. He also complained of reduce amount of urine but no dysurea. At that time patient's mental status also deteriorate. Then he was clinically diagnosed as post surgical meningitis and extensive cerebral extension of mucor was considered. CT scan of brain did not rule out new changes and CSF(cerebro spinal fluid) analysis showed neutrophilic leucocytosis.

With clinical possibilities of post surgical meningitis he was started Meropenem. Patient was clinically improved, became afebrile and mental status was normal. Then the patient was discharged on 22nd July 2015 and diagnosis was Post surgical acute meningitis, Rhinocerebral mucormycosis status post debridement.

After that the patient improved gradually for 5-6 days. On 30.7.15 the patient again deteriorated complaining of high grade fever and vomiting for several episodes and admitted to local hospital. But after that shifted to AKMMCH on 2.8.15 at 3.30AM. After admission suddenly patient's condition deteriorated. Massive convulsion occur and was shifted ICU(Intensive Care Unit). On 2.8.15 at 6 PM for low GCS(Glasgow Coma Scale) and severe convulsion patient was intubated on 6.15 $\mathrm{PM}$ and kept on mechanical ventilation(MV). He was diagnosed as a case of Type I respiratory failure(corrected by MV) with Meningo-encephalitis with $\mathrm{H} / \mathrm{O}$ Mucormycosis with Post surgical status with H/O FESS with DM (Diabetes mellitus) with Hypertension with Acute Kidney Injury with Left sided Haemothorax.

On admission, Complete blood count- $\mathrm{Hb} \%$ $7.2 \mathrm{gm} \%$, ESR-110 mm in first hour, Total count of WBC-5.71x109/L, and later on $14.16 \times 109 / \mathrm{L}, \mathrm{N}-$ $85 \%$, Platelet count-338x109/L, Hct $20.4 \%$, BT- 3 mins $30 \mathrm{sec}, \mathrm{CT}-8 \mathrm{mins} 15 \mathrm{sec}$, FDP- 13.36 $\mathrm{mg} / \mathrm{ml}$, D-Dimmer $1.03 \mathrm{mg} / \mathrm{L}$, Blood Urea $108 \mathrm{mg} / \mathrm{dl}$, S. Creatinine $2.7 \mathrm{mg} / \mathrm{dl}$, S. Electrolytes$\mathrm{Na}+132 \mathrm{mmol} / \mathrm{L}, \mathrm{K}+2.6 \mathrm{mmol} / \mathrm{L}, \mathrm{Cl}-98$ mmol/L, HCO3- 27 mmol/L, S. Calcium 8 mg/dl, 
S. Phosphate $3.15 \mathrm{mg} / \mathrm{dl}, \mathrm{S}$. Magnesium $1.36 \mathrm{mg} / \mathrm{dl}$, S. Bilirubin $0.60 \mathrm{mg} / \mathrm{dl}$, SGPT $26 \mathrm{U} / \mathrm{L}$, SGOT 49 U/L, S. Alkaline phosphatase 142U/L, STP $66.4 \mathrm{gm} / \mathrm{L}$, S. Albumin $31.8 \mathrm{~g} / \mathrm{L}, \mathrm{S}$. Uric acid 2.6 $\mathrm{mg} / \mathrm{dl}$, S. Ferritin $1756 \mathrm{ng} / \mathrm{ml}$, Total Cholesterol 107, LDL 70, HDL 27, TSH $1.47 \mathrm{mIU} / \mathrm{ml}$, HIV negative, $\mathrm{HBsAg}$ and Anti $\mathrm{HCV}$ negative, $\mathrm{ABG}$ Analysis- pH 7.405, PCO2 $31.7 \mathrm{~mm}$ of $\mathrm{Hg}, \mathrm{PO} 2$ $51.7 \mathrm{~mm}$ of $\mathrm{Hg}$, Hct $23.0 \%$, Urine $\mathrm{R} / \mathrm{E}$ Albumin ++ , Sugar nil, Pus 3-5/HPF, RBC nil, Urine $\mathrm{C} / \mathrm{S}$, Blood $\mathrm{C} / \mathrm{S}$, Tracheal aspirate $\mathrm{C} / \mathrm{S}$, CSF fluid $\mathrm{C} / \mathrm{S}$, Catheter tip $\mathrm{C} / \mathrm{S}$, Tip of Endotracheal tube $\mathrm{C} / \mathrm{S}$ all were of no growth.

CSF fluid study reviled Vol $2 \mathrm{ml}$, Protein $1.925 \mathrm{gm} / \mathrm{L}$, Sugar $34.2 \mathrm{mg} / \mathrm{dl}$, WBC $800 / \mathrm{cu} \mathrm{mm}, \mathrm{N}-80 \%$, L$20 \%$, Leishman's stain and Gram stain- no organism seen, ZN stain no AFB, Fungal stain not found, Bacterial Ag negative and negative of malignant cell.CSF for ADA 21.5 U/L, USG of KUB regionRight Kidney $15.1 \mathrm{~cm}$, Left Kidney $14.9 \mathrm{~cm}$. Comment- enlarged both kidneys with bilateral acute renal parenchymal disease. Left sided moderate pleural effusion. CT Scan of chest suggestive of Left sided haemorrhagic pleural effusion with collapse consolidation of left lower lobe.

Patient was started NG tube feeding. Meropenem, Vancomycin, Ceftriaxone were started in injectable form and in renal dose, anti convulsant and other symptomatic management given. Diabetes was controlled by short acting Insulin. Calcium channel blocker and Alpha receptor blocker used as antihypertensive. Frusemide was given as diuretics to increase urine output.

Examination of eyes: Left eye-Anterior segmentNAD, Pupil- reacting, Fundus- Diabetic retinopathy. Right eye- An-ophthalmic socket, enucleation. Eye lid- upper eye lid defected about $1.5 \mathrm{~cm} \times 1.5 \mathrm{~cm}$, both eyelid were adherent at the margin. Orbital surface- unhealthy tissue occupying whole of the surface with defect in the apex of the orbit which was $1 \mathrm{~cm} \times 1 \mathrm{~cm}$ continuous with the sphenoidal surface and advised for clean and dressing daily with anti-fungal eye drop and collection of orbital surface exudate for culture and sensitivity.

Tube thoracostomy done on 4.8.15 at left 6th intercostal space posterior to anterior axillary fold. 900 $\mathrm{ml}$ blood was evacuated and advice for gradual decompression, unclamping for 5-6 seconds and blood transfusion. Chest physiotherapy was started.

Gradually the patient's condition improved and was extubated from mechanical ventilation on 10.8.15. But 1 day after extubation patient developed laryngeal oedema and tracheostomy was done and maintained by high flow oxygen positive pressure ventilation. Thoracostomy tube was removed. After three days patient was send to cabin.
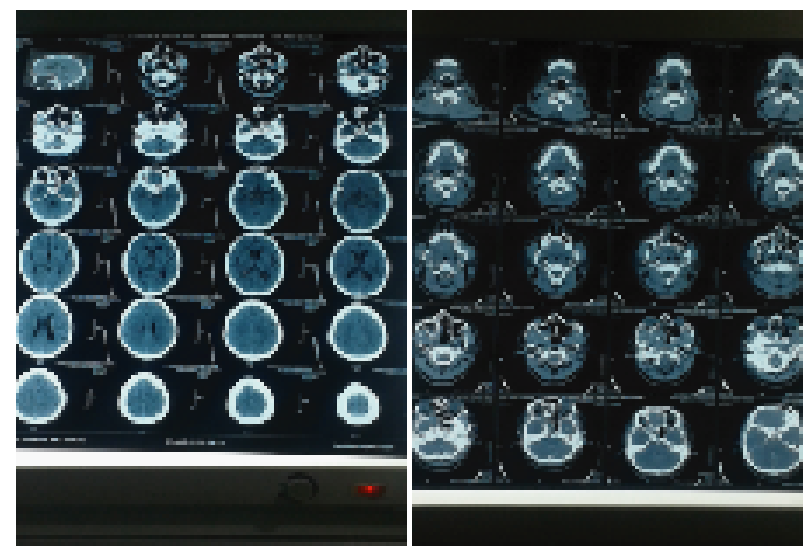

Fig: 1 MRI of Brain showed locally aggressive lesion consistent with mucormycosis in the right orbital apex, right maxillary ethmoidal sinus, right nasal cavity, pterygopontine fossa, lesser wings of sphenoid, optic foramina involving optic nerve, superior orbital fissure involving cranial nerves and extension into right middle cranial fossa involving meninges over cavernous sinus.of the

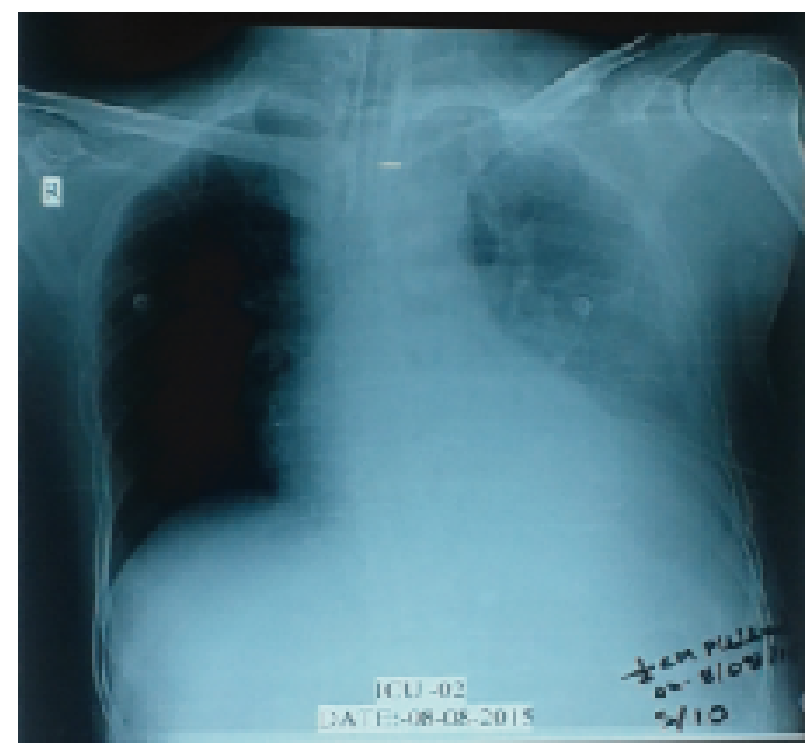

Fig: 2 X-Ray Chest P/A view showing Left sided Haemothorax with chest drain 
involved. 12,13,14 Amongst the paranasal sinuses, the most frequently involved are the maxillary and ethmoid sinuses.11 RCM is the most common form in patients with DKA(Diabetic ketoacidosis). ${ }^{16}$

Patients with rhinocerebral mucormycosis usually present with malaise, headache, facial pain, swelling and low grade fever. ${ }^{15}$ Rhino cerebral mucormycosis usually begins in the nasal mucosa or palate and extends to the paranasal sinuses, spreading via the angular, lacrimal, and ethmoid vessels as well as by direct extension from the sinuses into the retro-orbital region. Once fungal hyphae enter into the blood stream they can disseminate to other organs such as cerebrum or lungs which can be fatal for the patient. Mucor hyphae form thrombi within the blood vessels which reduce vascularity to the tissues and cause necrosis ${ }^{17}$. With orbital involvement, function of the cranial nerves II, III, IV and VI may be lost or impaired, with resultant proptosis, ptosis, papillary dilation, visual loss as well as periorbital cellulitis. Typical clinical presentation includes facial pain, presence of an irregular black eschar on the palatal or nasal mucosa and drainage of pus from the eye and nose $\mathrm{e}^{18}$.

In our case we have shown a male diabetic patient with poorly controlled diabetes mellitus who developed rhinocerebral mucormycosis with post surgical meningitis, haemothorax, type I respiratory failure with acute kidney injury. Ya-Chun Hsiao, Kun-Wu Tsan and Tao-Yeuan Wang, 2002, reported a female diabetic patient with poorly controlled blood sugar who developed rhinocerebral mucormycosis typically present with malaise, retro orbital headache, fever and occasionally dark blood tinged rhinorrhoea ${ }^{19}$. Vinay Vaidyanathan and Kishore Shetty, 2012, reported a series of 3 cases of rhinocerebral mucormycosis, in which 2 cases were successfully treated with Amphotericin B and one developed tubercular meningitis with obstructive hydrocephalus and the patient developed high morbid state ${ }^{20}$. In our case the patient developed meningitis 3 times and was successfully treated with broad spectrum antibiotics.

Kim et al. (2001) reported 4 cases of RM over a period of ten years, which were diagnosed postdental extractions. In all these 4 cases, the patients had an uncontrolled DM with two of them in a DKA state at the time of presentation ${ }^{15}$. Even though mucormycosis of the head and neck commonly
N Mahmood, F A Quraishi, S M H Shahid et al

occurs in the maxillofacial region, there are only few reported cases of RM in the mandible. ${ }^{6,17}$

Radiographically, maxillary mucormycosis typically shows opacification of the paranasal sinuses without fluid level, thickening of the sinus mucosa and bone distraction of the sinus walls [Figure: 7\& 8]. As some of these radiographic features may resemble sinusitis, McDonogh et al. warned that any diabetic patient in a ketoacidotic state presenting with clinical and radiographic features of rhinosinusitis should be suspected as having mucormycosis until proven otherwise ${ }^{21}$.

Definitive diagnosis of RM is usually obtained by a tissue biopsy which identifies the characteristic hyphae. ${ }^{18}$ Histologically, mucormycosis is characterised by extensive tissue necrosis and the presence of numerous, large $(5-30 \mu \mathrm{m})$, thinned-wall fungal hyphae, which are non-septate, branched at right angles and have a ribbon-like appearance [Figure: 9].

Successful management of rhinocerebral mucormycosis includes both medical and surgical modalities. The initial medical approach to mucormycosis consists of aggressive treatment of the underlying predisposing medical condition and the use of systemic antifungal agents. The use of amphotericin $\mathrm{B}$ in patients with mucormycosis has been widely published and accepted as the best form of treatment with an overall survival rate of up to $72 \% .15,16,18$ Based on recent published data, Spellberg et al. highlighted the recommended use of liposomal amphotericin B such as Am Bisome in the management of mucormycosis. ${ }^{22}$ Surgical management is crucially important and should involve early debridement of all infected and necrotic tissues. This may have to be repeated based on progression of the disease. In some cases, radical resection may be required, which can include partial or total maxillectomy, mandibulectomy and orbital exenteration. ${ }^{13,23}$ Reddy S Sujatha et al reported two cases of rhinocerebral mucormycosis in two uncontrolled diabetic patients with deep palatal perforation in one case and this was a rare and late occurrence. Both the patients were successfully treated with a combination of surgical debridement and systemic Amphotericin B administration. ${ }^{24}$

Hyperbaric oxygen(HBO)treatments should also be considered for those patients with aggressive 




Figure 3:

Axial CT of case 1 showing obliteration of the right maxillary sinus (black arrowhead) and soft tissue swelling over the right infraorbital region (white arrowhead)



Figure 4:

Coronal CT of case 1 showing obliteration of the right sinus causing blockage of the medial conchae and extension to the right ethmoid sinus (arrowhead)

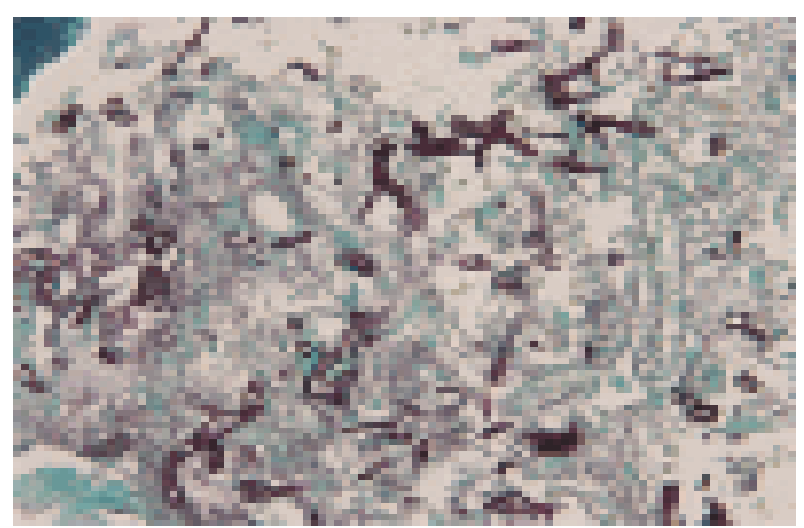

Figure 5:

Photomicrograph from retro-molar region showing elongated, broad, non-septate branching hyphae within the tissue and the bone marrow space (Gomori methenamine silver nitrate stain, original magnification_400)

\section{Discussion}

Mucormycosis is a fungal infection caused by a member of the family Mucoraceae.

Rhizopus, Mucor, Absidia are the most common isolated from patients with mucormycosis. Rhizopus is responsible for $60 \%$ of all cases of mucormycosis, and $90 \%$ of rhinocerebral mucormycosis 6,7 . The fungi are found in soil, bread, mold, rotten fruits and vegetables. Though mucormycosis is ubiquitous and grows rapidly, it seldom strikes in immunologically competent patients. Therefore, if an infection with mucormycosis occurs, it usually indicates a serious underlying medical condition. The risk factors for developing rhinocerebral mucormycosis include severe burn, acquired immune deficiency syndrome (AIDS), immunosuppressive medications, leukemia, diabetes mellitus, and organ transplantation ${ }^{8}$. About $70 \%$ to $80 \%$ of these patients have diabetes mellitus. As is reported in our case, most diabetics who develop rhinocerebral mucormycosis (RCM) are in poor metabolic control with complicating ketoacidosis ${ }^{9}$. It is suggested that fungal organism grows in ketotic patients because acidosis disrupts iron binding to transferring and the result increases in free iron then promotes growth of the fungus. At the same time, high blood sugar level may also alter the immunologic capability to resist mucormycosis through reduction of $\mathrm{WBC}$ chemotaxis and the ability of macrophages ${ }^{10}$.

Baker introduced the term ''rhinocerebral mucormycosis"' as a malignant form of cephalic mucormycosis with a high mortality rate. In 1958 Chick et al, demonstrated the effectiveness of amphotericin B. Today, the most commonly chosen therapy consists of the treatment of predisposing diseases, radical surgery, and systemic antifungal medication, especially with amphotericin B. Despite these aggressive therapeutic measures the mortality continues to be very high. ${ }^{1}$

There are at least six clinical forms of mucormycosis: rhinocerebral, pulmonary, gastrointestinal, cutaneous, central nervous system, disseminated and miscellaneous, occurring at specific organ sites e.g. kidney and liver. ${ }^{11,12,15}$ The term rhinocerebral mucormycosis (RCM) should be used when the paranasal sinuses, orbit and brain are 
infections. A few studies have shown that HBO has direct in vitro fungistatic activity and reduce tissue hypoxia, which may reverse the hypoxic acidosis that helps the fungi to proliferate 25 . Blitzer and Lawson found that in their review of 170 cases of RCM, 63\% of untreated diabetics died as compared with $17 \%$ mortality rate when therapy included aggressive surgery and amphotericin B administration9. The importance of surgery is pronounced when no surgical treatment or only biopsy was performed the mortality rate is as high as $58 \%^{9}$.

In our reported case, the management was started early in the course of disease and involved aggressive local debridement with repeated antiseptic packing until the wound healed with healthy granulation tissue. In addition, the correction of the underlying medical condition, along with the use of liposomal amphotericin B therapy, resulted in good control of the disease with a favourable outcome in initial stage. Post surgical complications were managed accordingly. Rehabilitation or closure of the orbital socket and maxillary hole can be done surgically or by construction of a prosthetic appliance.

\section{Conclusion}

Mucormycosis is a rapidly progressive disease with a fulminant course and fatal outcome unless diagnosed early and treated rapidly. Prognosis is dependent on multiple factors and early initiation of treatment is an important element. A multidisciplinary approach consisting of neurologist, dental specialists, ENT surgeons, ophthalmologists and nephrologist is critical in successful management of a patient with mucormycosis.

\section{Acknowledgement}

The author would like to thank the patient for giving the permission to report the case for the journal and the medical officers of ICU for their contribution.

\section{Conflict of interest: no}

\section{References}

1. Scheckenbach K, Cornely O, Hoffmann TK, Engers R, Bier H, Chaker A, et al. Emerging therapeutic options in fulminant invasive rhinocerebral mucormycosis. Auris Nasus Larynx 2010; 37: 322-8. [PubMed]
2. Cheema SA, Amin F. Five cases of rhinocerebral mucormycosis. Br J Oral Maxillofac Surg 2007; 45: 161-2.[PubMed]

3. Rangel-Guerra RA, Martínez HR, Sáenz C, BosquesPadilla F, Estrada-Bellmann I. Rhinocerebral and systemic mucormycosis. Clinical experience with 36 cases. J Neurolog Sci 1996; 143: 19-30.

4. Dökmetas HS, Canbay E, Yilmaz S, Elaldi N, Topalkara A, Oztoprak I, et al. Diabetic ketoacidosis and rhinoorbital mucormycosis. Diabetes Res Clin Pract 2002; 57: 139-42.

5. Ogawa T, Takezawa K, Tojima I, Shibayama M, Kouzaki H, Ishida M, et al. Successful treatment of rhino-orbital mucormycosis by a new combination therapy with liposomal amphotericin B and micafungin. Auris Nasus Larynx 2012; 39: 224-8.

6. Brown OE, Finn R. Mucormycosis of the mandible. J. Oral Maxillofac Surg1986; 44: 132-6.

7. Galetta SL, Wulc AE, Goldberg HI, et al. Rinocerebral mucormycosis : management and survival after carotid occlusion. Ann Neurol 1990; 28: $103-7$

8. Lee EJ, Lee MY, Hung YC, et al. Orbital rhinocerebral mucormycosis associated with diabetic ketoacidosis report of survival of a 10year-old boy. J Formos Med Assoc. 1998; 97: 720-3.

9. Ricardo AR, Hector RM, Corando S, et al. Rhinocerebral and systemic mucormycosis clinical experience with 36 cases. J Neurol Scien 1996; 143: 19-30.

10. Kenton O, James E. Infectious emergencies in patients with diabetes mellitus. Med Clin North Am 1995; 79: 53-77.

11. McNulty JS. Rhinocerebral Mucormycosis: Predisposing Factors. Laryngoscope. 1982; 92: 1140-1144. [PubMed]

12. deShazo RD, O'Brien M, Chapin K, Soto-Aguilar M, Gardner L, Swain R. A new classification and diagnostic criteria for invasive fungal sinusitis. Arch Otolaryngol Head Neck Surg. 1997; 123: 1181-1188. [PubMed]

13. Tryfon S, Stanopoulos I, Kakavelas E, Nikolaidous A, Kioumis I. Rhinocerebral Mucormycosis in a Patient with latent diabetes mellitus: A Case Report. J Oral Maxillofac Surg. 2002; 60: 328-330. [PubMed] 
14. Peterson KL, Wang M, Canalis RF. Rhinocerebral Mucormycosis: Evaluation of the Disease and Treatment Options. Laryngoscope. 1997; 107: 855-861. [PubMed]

15. Kim J, Fortson JK, Cook HE. A Fatal Outcome from Rhinocerebral Mucormycosis after Dental Extractions: A Case Report. J Oral Maxillofac Surg. 2001; 59: 693-697. [PubMed]

16. Sugar AM. Mucormycosis. Clin Infect Dis. 1992; 14: 126-129. [PubMed]

17. Salisbury PL, Caloss R Jr, Cruz JM, Powell BL, Cole R, Kohut RI.Mucormycosis of the mandible after dental extractions in a patient with acute myelogenous leukemia. Oral Surg Oral Med Oral Pathol Oral Radiol Endod. 1997; 83: 340-4.

18. Tugsel Z, Sezer B, Akalin T. Facial swelling and palatal ulceration in a diabetic patient. Oral Surg Oral Med Oral Pathol Oral Radiol Endod. 2004; 98: 630-6.

19. Ya-Chun Hsiao, Kun-Wu Tsan, Tao-Yeuan Wang. Rhinocerebral Mucormycosis in Diabetes? A Case Report. J Intern Med Taiwan 2002; 13: 160-164.
20. Vinay Vaidyanathan, Kishore Shetty. Rhinocerebral Mucormycosis:A series of 3 cases. Ann Trop Med Public Health 2012; 5: 591-3.

21. McDonogh $M$, Human $P$, Odendaal W. Mucorsinisitis in diabetes. S Afr Med J. 1985; 67: 78-81. [PubMed]

22. Spellberg B, Edwards J, Ibrahim A. Novel perspectives on mucormycosis: pathophysiology, presentation, and management. Clin Microbiol Rev. 2005; 18: 556-569. [PMC free article] [PubMed]

23. Ribeiro NNF, Cousin GCS, Wilson GE, Butterworth DM, Woodwards RTM. Lethal invasive mucormycosis: Case report and recommendation for treatment. Int $\mathrm{J}$ Oral Maxillofac Surg. 2001; 30: 156-159. [PubMed]

24. Reddy S. Sujatha, N. Rakesh, Jatti Deepa, Lanjekar Ashish, Bijjal Shridevi. Rhino cerebral mucormycosis. A report of two cases and review of literature. J Clin Exp Dent. 2011; 3(3): 256-60.

25. Kajs-Wyllie M. Hyperbaric oxygen therapy for rhinocerebral fungal infection. J Neurosci Nurs 1995; $27: 174$. 